\title{
A step closer in the management of uncontrolled neuropathic pain: gabapentin-nortriptyline combination
}

\author{
Manish Nilkanth Maladkar ${ }^{1 *}$, Chitra Mohan Tekchandani², Raveena Kanyalal Bajaj²
}

\author{
${ }^{1}$ Medical and Regulatory Affairs, Scientific Department, Aristo Pharmaceuticals Private Limited, Mumbai, \\ Maharashtra, India \\ ${ }^{2}$ Medical, Scientific Department, Aristo Pharmaceuticals Private Limited, Mumbai, Maharashtra, India
}

Received: 11 August 2020

Accepted: 17 September 2020

\author{
*Correspondence: \\ Dr. Manish Maladkar, \\ E-mail: scientific@aristopharma.org
}

Copyright: () the author(s), publisher and licensee Medip Academy. This is an open-access article distributed under the terms of the Creative Commons Attribution Non-Commercial License, which permits unrestricted non-commercial use, distribution, and reproduction in any medium, provided the original work is properly cited.

\begin{abstract}
Neuropathic pain is extremely distressing as it significantly hampers the performance of daily activities and impedes overall quality of life. A large number of illnesses are linked with neuropathic pain like radiculopathy, sciatica, diabetes, herpes infection, spinal injury, stroke, etc. Treating neuropathic pain is challenging as response to the treatment is at times uncertain and associated with undesirable side effects. Uncontrolled neuropathic pain is observed in large group of patients receiving current treatment regimen. Almost half of the neuropathic pain patients need more than two drugs for pain relief. Various clinical trials have demonstrated better efficacy of combination therapy in reducing pain than monotherapy. Utilizing drug combination with different mechanism of action or acting on two different sites has demonstrated superior analgesic effect in painful neuropathic condition. The first tier agents recommended by various guidelines are calcium channel alpha-2-delta ligands and tricyclic antidepressants that act on different pain pathways. A clinical trial has shown enhanced pain relief with no increase in side effects with the administration of gabapentin and nortriptyline combination in patients suffering from neuropathic pain. Moreover, symptoms associated with neuropathic pain like sleep disturbances, altered mood, decreased walking ability, depression and quality of life were also significantly improved. Hence, combination therapy of gabapentin and nortriptyline offers a pivotal modality in the management of uncontrolled neuropathic pain.
\end{abstract}

Keywords: Neuropathic pain, Calcium channel alpha-2-delta ligand, Tricyclic antidepressants, Gabapentin, Nortriptyline, Combination therapy

\section{INTRODUCTION}

The electrochemical signaling in neurons, though a highly complex process, plays a key role in physiological homeostasis. Any damage to this neuronal signaling network is associated with several adverse repercussions. Neuropathy is one such consequence that occurs due to nerve injury or compression that affects the normal activity of the nervous system. ${ }^{1}$ The symptoms associated with neuropathy include burning, tingling, numbness, pain, difficulty in walking, pins and needles, reduced sensation of touch or oversensitivity, etc. Among these, pain is one of the most common and troublesome symptoms experienced by the patient suffering from neuropathy. According to the International association for the study of pain (IASP), neuropathic pain is defined as 'pain arising as a direct consequence of a lesion or disease affecting the somatosensory system. ${ }^{2}$ Neuropathic pain is witnessed in varied clinical conditions, such as radiculopathy, sciatica, diabetes, spinal cord injury, post-surgery, herpes infection, stroke, fibromyalgia, etc. ${ }^{4}$ It may be classified as peripheral neuropathic pain, central neuropathic pain and 
mixed neuropathic pain depending upon the affected area of the neurons. Pain associated with neuropathy can also be classified depending on the duration as acute and chronic in nature, the latter is observed in majority of the patients. ${ }^{4}$ It has substantial impact on the patients' overall quality of life thus, impeding their daily activities. In addition to neuropathic pain, the patient experiences psychological variations as well, like anxiety and depression. ${ }^{5}$ Taking together, neuropathic pain is a cluster of different conditions which has a significant unfavorable effect on patients overall health.

\section{NEUROPATHIC PAIN MANAGEMENT}

Neuropathic pain is one of the difficult medical ailments to treat and the key aim is reduction of pain. Neuropathic pain management necessitates an interdisciplinary methodology that embraces pharmacological as well as non-pharmacological interventions. Physiotherapy is a part of the management of most chronic pain conditions which aims to improve the patients' physical activity rather than to reduce pain. Since many years several drugs are available in the armamentarium of neuropathic pain management. Numerous systematic reviews have abridged the available evidences for the treatment of neuropathic pain and guidelines have been published for an organized treatment approach. Drug classes, including tricyclic antidepressants, calcium channel alpha-2-delta ligands, opioid analgesics, topical lidocaine and capsaicin have demonstrated consistent efficacy in several clinical studies. Guidelines like IASP, European Federation of Neurological Societies, Canadian Pain Society, and Mayo Clinic Proceedings recommend calcium channel alpha-2delta ligands, tricyclic antidepressants and lidocaine patches as first line agents for the treatment of neuropathic pain..$^{6-9}$ In focal neuropathic pain observed in postherpetic neuralgia, the latter may be considered for the treatment. ${ }^{10,11}$ Additionally, carbamazepine or oxcarbazepine from benzodiazepine class is the drug of choice for the treatment of trigeminal neuralgia.

Calcium channel alpha-2-delta ligands have been used extensively as a primary agent in treating neuropathic pain. ${ }^{12}$ In particular, gabapentin and pregabalin have been supported by large number of evidences for their efficacy and safety in the management of neuropathic pain. The mechanism involves blocking of the alpha-2-delta subunit of calcium channel in the ascending pain pathway, limiting the release of excitatory neurotransmitter glutamate and substance $\mathrm{P}$ responsible for pain transmission. ${ }^{13,14}$ The American diabetes association (ADA), American association of clinical endocrinologists (AACE) and American academy of neurology (AAN) recommend gabapentin for management of diabetic neuropathy. ${ }^{15-17}$ Moreover, gabapentin and pregabalin have demonstrated efficacy in reducing anxiety associated with neuropathy which improves the therapeutic outcome. ${ }^{18,19}$ Antidepressants are used in confronting neuropathic pain from a long time and various clinical trials have recognized their role as analgesics in neuropathy. Selective serotonin reuptake inhibitors like fluoxetine and paroxetine as well as serotonin norepinephrine reuptake inhibitors like duloxetine and milnacipran have shown efficacy in treating neuropathic pain and could be considered if the first line drugs fail. ${ }^{20}$ The widely used class of drugs, Tricyclic antidepressants (TCAs) has been recommended by ADA for the management of diabetic neuropathy. ${ }^{15}$ TCAs can be further classified into tertiary amines (amitriptyline and imipramine) and secondary amines (nortriptyline and desipramine). These drugs block the reuptake of serotonin and norepinephrine thereby increase the availability of these inhibitory neurotransmitters in the descending pain pathway which assists in reducing the neuropathic pain. The secondary amines like nortriptyline are better tolerated than tertiary amines in terms of sedation and other side effects. ${ }^{21}$ The antidepressant effect is beneficial in chronic neuropathic pain conditions as depression is a common comorbidity. ${ }^{22}$

Opioid analgesics along with tramadol constitute second line agents for neuropathic pain and third line agents include bupropion, citalopram, lamotrigine, topiramate, topical low concentration drugs such as capsaicin, dextromethorphan, memantine and mexiletine. Neuropathic pain agents have been tested in one or different types of neuropathic pain settings and extrapolation of efficacy to different types of neuropathic pain is reasonable and clinically necessary.

\section{UNCONTROLLED NEUROPATHIC PAIN}

In spite of availability of numerous treatment options, neuropathic pain management is still a challenge due to several reasons, namely erratic response to the drug, dose titration leading to intolerable side effects and unaddressed comorbid psychological condition. Thus, moderate to severe level of pain prevails that contributes to the growing incidence of patients suffering from uncontrolled neuropathic pain. It refers to pain score greater than or equal to 4 in the DN4 (Douleur Neuropathique 4) questionnaire. $^{23-25}$

Neuropathic pain is associated with the psychological comorbidities in major number of patients like sleep disturbances, depression and anxiety. ${ }^{26}$ The pain and immobility because of neuropathy makes the individual anxious regarding the effectiveness of the treatment or depressed as the patient is not able to perform the normal day to day activities. Additionally, the social life of a patient gets disturbed due to many restrictions which are again stressful for the patient. The management of neuropathic pain involves certain medication linked with higher degree of side effects which does not allow the uptitration of dose for further reduction of pain. Moreover, addition of drug from another class with overlapping side effects sounds unacceptable. These factors make the therapeutic approach for neuropathic pain unassured or incomplete. Abundant clinical trials have shown the efficacy of suggested agents in different neuropathic conditions including diabetic neuropathy, postherpetic 
neuralgia, fibromyalgia, spinal injury, lumbar stenosis, low back pain. However, the applicability of result obtained from a drug for a particular condition to other neuropathic pain disorders is difficult to determine as some types of neuropathic pain patients may respond differently. Additionally, the association with psychological comorbidities remains unaddressed while tackling neuropathic pain. Thus, uncontrolled neuropathic pain is a common phenomenon associated with neuropathic pain sufferers with an intense impact on patient well-being. A definite necessity exists for improving the management of uncontrolled neuropathic pain, which should not be restricted to reduce pain but also should be extended to the management of psychological symptoms.

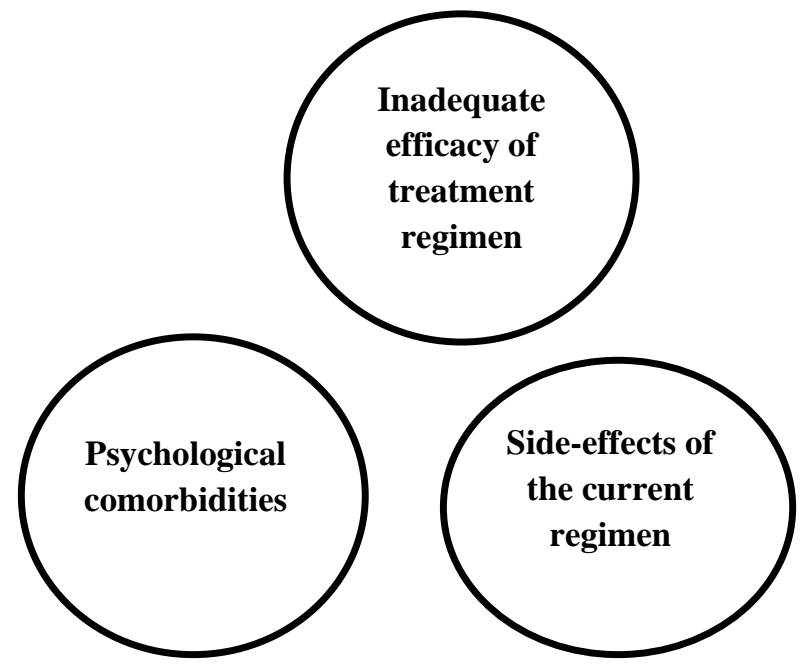

Figure 1: Reasons of uncontrolled neuropathic pain.

\section{COMBINATION THERAPY IN THE MANAGEMENT OF UNCONTROLLED NEUROPATHIC PAIN}

As discussed earlier, current treatment regimen does not provide adequate pain relief in many of the neuropathic pain sufferers. Emphasis for combining two agents is conversed in many literatures and could be a plausible choice for satisfactory pain relief. ${ }^{24,27,28}$ Combination of drugs with different modes of action may be beneficial in tackling neuropathic pain in a more effective manner. ${ }^{29}$ Various trials have demonstrated a potential role of combination therapy furnishing the base for the employment of dual drug therapy as a cornerstone in the management of uncontrolled neuropathic pain. Additionally, it is reported that $45 \%$ of neuropathic pain patients require two or more drugs for their pain relief. ${ }^{30}$

Several trials have reported that combination of medications substantially reduces more pain than monotherapy. Gabapentin in combination with morphine demonstrated better analgesia at lower doses than either agent administered alone. ${ }^{31}$ The combination of gabapentin and nortriptyline markedly improved pain in patients suffering from chronic orchialgia. ${ }^{32}$ Gabapentin and oxycodone combination is superior to gabapentin alone in diabetic neuropathy. ${ }^{33}$ Oral gabapentin and ketamine infusion when administered together produces greater pain relief when compared to oral gabapentin alone. ${ }^{34}$ Duloxetine and pregabalin combination is safe, effective and well tolerated in patients suffering from diabetic neuropathic pain. ${ }^{35}$ Epalrestat and methylcobalamin combination revealed faster and better neuropathic pain reduction associated with diabetes than epalrestat monotherapy. ${ }^{36}$ Tramadol and acetaminophen combination is more effective and well tolerated than placebo in the management of painful diabetic neuropathy. ${ }^{37}$ Faster analgesia is achieved with combination of capsaicin and doxepin topical application as compared to a single agent. ${ }^{38}$

\section{GABAPENTIN \\ AND \\ NORTRIPTYLINE COMBINATION}

Clinical study carried out in patients suffering from neuropathic pain evaluated and demonstrated that gabapentin and nortriptyline combination is an efficacious and safe therapy in the management of neuropathic pain. Gabapentin when given in combination with nortriptyline significantly reduces neuropathic pain than either of the single agent. The pain score is found to be significantly reduced from the second day of administering the combination therapy. There is almost $18 \%$ greater pain reduction with gabapentin and nortriptyline combination therapy as compared to monotherapy. Moreover, the combination improves depression, mood, sleep disturbances, walking ability and overall health related quality of life. Gabapentin and nortriptyline, both have some set of common side effects like somnolence and dizziness but it is demonstrated that the intensity of side effects do not elevate and are comparable with monotherapy of both the drugs when given in combination. ${ }^{39}$ On a wider note, managing uncontrolled neuropathic pain requires a combination of drugs which enhances or complements the action of other drug and improves the tolerability profile. Focusing on a potential combination of calcium channel alpha-2-delta ligands like gabapentin and TCAs like nortriptyline acting on complementary pathways provide enhanced pain relief without increasing the side effects which further enhances the tolerability of the regimen. Importantly, the combination of gabapentin and nortriptyline is cited by the National institute of health and care excellence (NICE) guidelines while considering the treatment strategies. ${ }^{40}$ The combination therapy of gabapentin with tricyclic antidepressants is highly recommended (highest grading) for the management of neuropathic pain by Danish expert committee based on scientific evidence and clinical experience. $^{41}$ Moreover, a consensus statement from Indian experts also recommends this combination. ${ }^{42}$ Fixed dose combinations especially of gabapentin with nortriptyline can be synergistic and improve treatment adherence, especially in Indian setting where compliance to therapy is generally poor. 
The combination of gabapentin and nortriptyline has a strong peer reviewed backing with several authors stating about the advantages of combination.

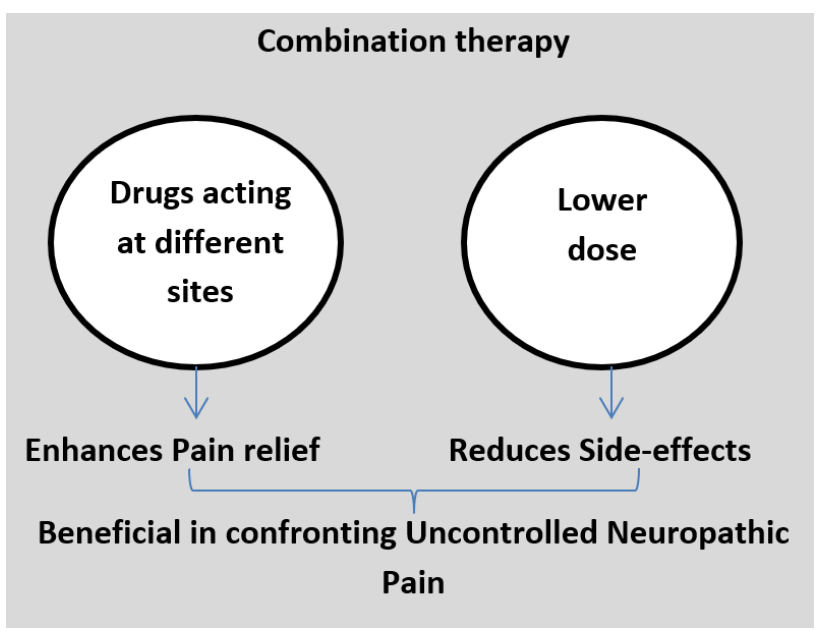

Figure 2: Advantages of combination therapy in managing uncontrolled neuropathic pain.

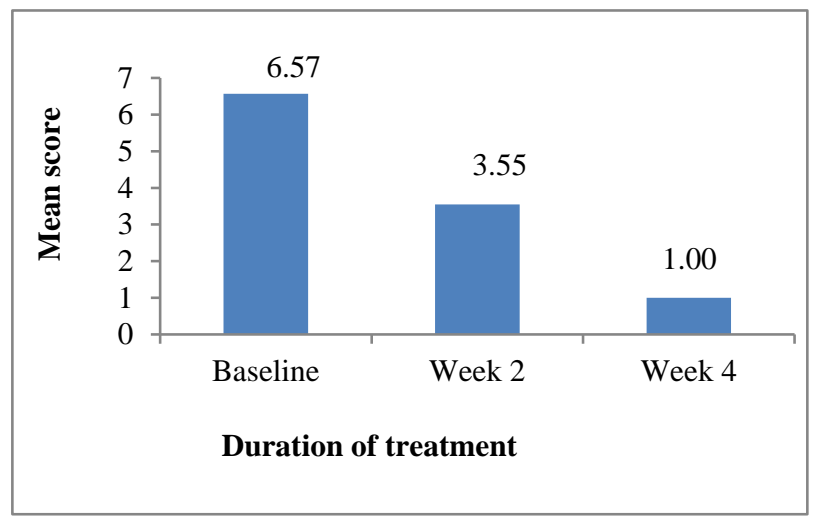

Figure 3: Mean pain intensity score.

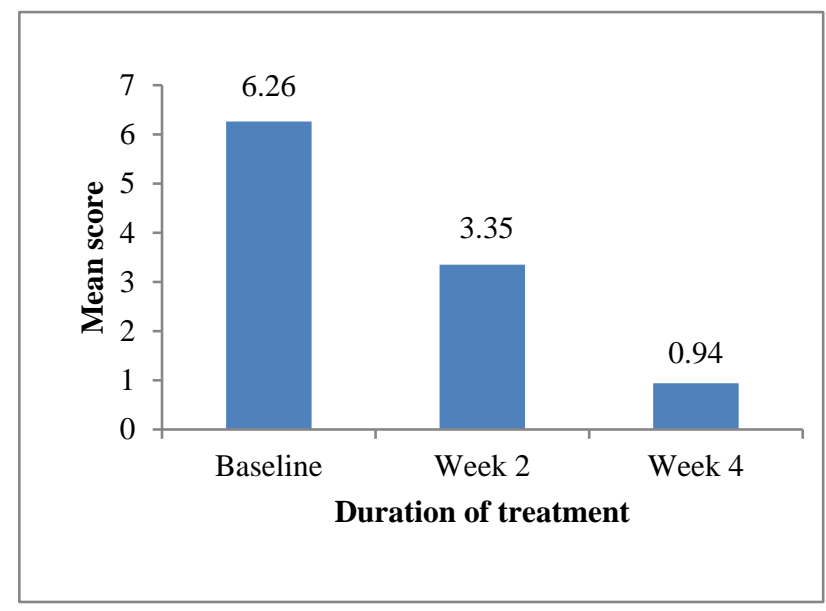

Figure 4: Mean general activity score.

Combination therapy decreases pain as well as sleep interference without elevating the side effects. ${ }^{43}$ The pain intensity with combination therapy of gabapentin and nortriptyline is lower than with either of the single agent. ${ }^{44}$ Superior results have been obtained with combined treatment of gabapentin and nortriptyline than each agent alone in pain control; these agents should be strongly considered in combination in patients experiencing inadequate relief on monotherapy. ${ }^{45,46}$

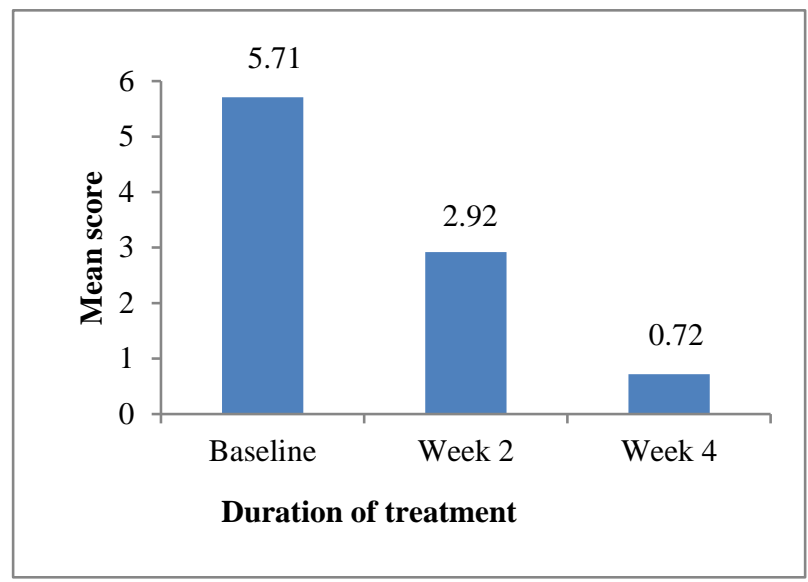

Figure 5: Mood score.

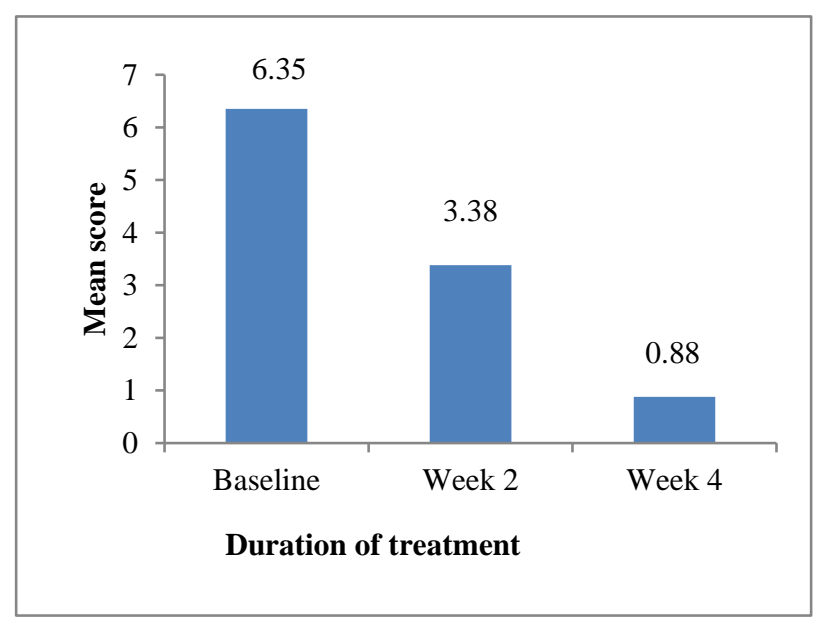

Figure 6: Sleep score.

A post-marketing surveillance was conducted in 494 Indian diabetic neuropathy patients treated with gabapentin $300 \mathrm{mg}$ - nortriptyline $10 \mathrm{mg}$ combination (Gabaneuron $\mathrm{NT}^{\mathrm{TM}}$ ). Average age of the patients was 43.47 years and mean weight was $65.21 \mathrm{~kg}$. Physical examination like temperature, pulse rate, respiratory rate and blood pressure were within normal limits at baseline.

As per the data, there was significant improvement in symptoms like fatigue, pain in lower legs, weakness, numbness, tingling, burning pain, severe pain in low back region. Improvement in symptoms was reflected in improvement in efficacy parameters- mean pain intensity score, mean general activity score, mood score and sleep score. Mean pain intensity score (as shown in the figure 3) decreased from 6.57 to 1.00 on treatment with gabapentinnortriptyline combination for 4 weeks. Mean general activity score (Figure 4) significantly reduced from 
baseline 6.26 to 0.94 at the end of 4 weeks. Similarly, mood score (figure 5) and sleep score (figure 6) also decreased from 5.71 to 0.72 and 6.35 to 0.88 respectively at the end of 4 weeks.

The treatment was found to be well-tolerated; $3.6 \%$ patients experienced adverse events including nausea, vomiting and drowsiness and most of these events resolved without any intervention.

\section{CONCLUSION}

Uncontrolled neuropathic pain has debilitating outcomes with significant negative impact on the quality of life. Clinical trials have demonstrated enhanced pain relief with combination therapy than with either drug alone. Gabapentin with nortriptyline seems a potential therapeutic approach in management of uncontrolled neuropathic pain. This fixed dose combination can improve treatment adherence, especially in Indian settings where compliance to therapy is generally poor.

\section{Funding: No funding sources \\ Conflict of interest: None declared \\ Ethical approval: Not required}

\section{REFERENCES}

1. England DJ, Arthur AK. Periph Neurop. Lancet. 2004;363 2151-61.

2. Jensen, Troels S. A new definition of neuropathic pain. Pain. 2011;152(10):2204-5.

3. Scadding, John, Koltzenburg M. Neuropathic pain. Advan Clin Neuro Rehab. 2003;3:8-14.

4. The American Academy of Pain Medicine. AAPM facts and figures on pain. AAPM Facts and Figures on Pain. 2013. Accessed on 10 July, 2020.

5. Gormsen, Lise. Depression, anxiety, health related quality of life and pain in patients with chronic fibromyalgia and neuropathic pain. Eur J Pain. 2010;14(2):127-e1.

6. Ballantyne, Jane C. Pharmacological management of neuropathic pain. Pain: Clinical Updates. 2010. Accessed on 10 July, 2020.

7. Attal N. EFNS guidelines on the pharmacological treatment of neuropathic pain: 2010 revision. Eur J Neurol. 2010;17(9):1113-e88.

8. Moulin DE. Pharmacological management of chronic neuropathic pain: revised consensus statement from the Canadian Pain Society. Pain Research and Management. 2014;19(6):328-35.

9. Dworkin, Robert H. Recommendations for the pharmacological management of neuropathic pain: an overview and literature update. Mayo Clinic Proceedings. 2010;85(3):S3-14.

10. Davies, Pamela S, Bradley S. Galer. Review of lidocaine patch $5 \%$ studies in the treatment of postherpetic neuralgia. Drugs. 2004;64(9):937-47.

11. Meier T. Reduction of chronic pain for nonpostherpetic peripheral neuropathies after topical treatment with a lidocaine patch. Schmerz (Berlin, Germany). 2004;18(3):172-8.

12. Wiffen, Philip J. Antiepileptic drugs for neuropathic pain and fibromyalgia-an overview of Cochrane reviews. Cochrane Database Syst Rev. 2013;11(11):CD010567.

13. Raison VMCL. Neurobiology of depression, fibromyalgia and neuropathic pain. Frontiers in Bioscience 14. 2009;5291-338.

14. Taylor, Charles P. Mechanisms of analgesia by gabapentin and pregabalin-Calcium channel $\alpha 2-\delta$ [Cava2- $\delta$ ] ligands. Pain. 2009;142(1-2):13-6.

15. Pop-Busui R, Boulton AJ, Feldman EL. Diabetic Neuropathy: A Position Statement by the American Diabetes Association. Diabetes Care. 2017;40(1):136-54.

16. Handelsman Y, Bloomgarden ZT, Grunberger G, Umpierrez G, Zimmerman RS, Bailey TS, et al. American Association of Clinical Endocrinologists and American College of Endocrinology- clinical practice guidelines for developing a diabetes mellitus comprehensive care plan-2015. Endocr Pract. 2015;21(1):1-87.

17. Bril V, England J, Franklin GM, Backonja M, Cohen J, Del Toro D, et al. Evidence based guideline: treatment of painful diabetic neuropathy: report of the American Academy of Neurology, the American Association of Neuromuscular and Electrodiagnostic Medicine. Amer Acad Phys Med Rehab Neurol. 2011;76(20):1758-65.

18. Finnerup, Nanna B, Troels S. Jensen. Clinical use of pregabalin in the management of central neuropathic pain. Neuropsychiatric disease and treatment. 2007;3(6):885-91.

19. Perret, Danielle, Luo DZ. Targeting voltage-gated calcium channels for neuropathic pain management. Neurotherapeutics. 2009;6(4):679-92.

20. Lee, Yee-Chi, Chen PP. A review of SSRIs and SNRIs in neuropathic pain. Expert opinion on pharmacotherapy. 2010;11(17):2813-25.

21. Beydoun A. Symptomatic treatment of neuropathic pain: a focus on the role of anticonvulsants. Medscape CME Circle Lecture. 2001. Accessed on 11 July, 2020.

22. Raison VMCL. Neurobiology of depression, fibromyalgia and neuropathic pain. Frontiers in Bioscience 14. 2009;5291-338.

23. de la Calle, José-Luis. Add-on treatment with pregabalin for patients with uncontrolled neuropathic pain who have been referred to pain clinics. Clinical drug investigation. 2014;34(12):833-44.

24. Harden, Norman, Cohen M. Unmet needs in the management of neuropathic pain. Journal of pain and symptom management. 2003;25(5):S12-7.

25. José A. Clinical characteristics, patient-reported outcomes, and previous therapeutic management of patients with uncontrolled neuropathic pain referred to pain clinics. Pain research and treatment. 2014;2014:518716. 
26. Turk, Dennis C. Assessment and treatment of psychosocial comorbidities in patients with neuropathic pain. Mayo Clinic Proceedings. 2010;85(3):S42-50.

27. Dworkin, Robert H. Recommendations for the pharmacological management of neuropathic pain: an overview and literature update. Mayo Clinic Proceedings. 2010;85(3):S3-14.

28. Ballantyne, Jane C, Carwood CM, Giamberardino MA. Pharmacological management of neuropathic pain. Pain: Clinical Updates. 2010. Accessed on 10 July, 2020.

29. Attal, Nadine. EFNS guidelines on pharmacological treatment of neuropathic pain. European Journal of Neurology. 2006;13(11):1153-69.

30. Chaparro, Enrique L. Combination pharmacotherapy for the treatment of neuropathic pain in adults. Cochrane Database Syst Rev 7. 2012.2012(7):CD008943.

31. Gilron, Ian. Morphine, gabapentin, or their combination for neuropathic pain. New England Journal of Medicine. 2005;352(13):1324-34.

32. Sinclair, Andrew M, Miller B, Lee LK. Chronic orchialgia: consider gabapentin or nortriptyline before considering surgery. International journal of urology. 2007;14(7):622-5.

33. Hanna, Magdi, O'Brien C, Wilson MC. Prolongedrelease oxycodone enhances the effects of existing gabapentin therapy in painful diabetic neuropathy patients. European Journal of Pain. 2008;12(6):80413.

34. Amr, Yasser Mohamed. Multi-day low dose ketamine infusion as adjuvant to oral gabapentin in spinal cord injury related chronic pain: a prospective, randomized, double blind trial. Pain Physician. 2010;13(3):245-9.

35. Tesfaye, Solomon. Duloxetine and pregabalin: highdose monotherapy or their combination? The COMBO-DN study-a multinational, randomized, double-blind, parallel-group study in patients with diabetic peripheral neuropathic pain. PAIN®. 2013;154(12):2616-25.

36. Maladkar, Manish. Evaluation of efficacy and safety of epalrestat and epalrestat in combination with methylcobalamin in patients with diabetic neuropathy in a randomized, comparative trial. Journal of Diabetes mellitus. 2013;3:22-26.

37. Freeman, Roy. Randomized study of tramadol/acetaminophen versus placebo in painful diabetic peripheral neuropathy. Current medical research and opinion. 2007;23(1):147-61.

38. McCleane, Gary. Topical application of doxepin hydrochloride, capsaicin and a combination of both produces analgesia in chronic human neuropathic pain: a randomized, double-blind, placebo-controlled study. British journal of clinical pharmacology. 2000;49(6):574-9.

39. Gilron, Ian. Nortriptyline and gabapentin, alone and in combination for neuropathic pain: a double-blind, randomised controlled crossover trial. The Lancet. 2009;374(9697):1252-61.

40. Longson D. Neuropathic pain-pharmacological management: the pharmacological management of neuropathic pain in adults in non-specialist settings. NICE clinical guideline. 2013;173. Accessed on 10 July, 2020.

41. Holbech. Combination treatment of neuropathic pain: Danish expert recommendations based on a Delphi process. Journal of Pain Research. 2017:10:1467-75.

42. Saxena AK, Jain P, Dureja GP, Venkitachalam A, Goswami S, Usmani $\mathrm{H}$, et al. Pharmacological management of neuropathic pain in India: A consensus statement from Indian experts. Indian J Pain. 2018;32:132-44.

43. Gandey A. Combination Gabapentin and Nortriptyline May Ease Neuropathic Pain. Medscape Medical News. 2009. Accessed on 10 July, 2020.

44. Soloway B. Gabapentin-Nortriptyline Combination Therapy for Neuropathic Pain. NEJM Journal watch. 2009. Accessed on 11 July, 2020.

45. Gilron I, Bailey J, Dongsheng T. Combined treatment with gabapentin and nortriptyline improves pain control in peripheral neuropathy more than either agent alone. JCOM 16. 2009;16(12):544-45.

46. O'Connor, Alec B. Study finds that the combination gabapentin plus nortriptyline reduces neuropathic pain more than either drug alone. Evidence-based medicine. 2010;15(2):45.

Cite this article as: Maladkar MN, Tekchandani CM, Bajaj RK. A step closer in the management of uncontrolled neuropathic pain: gabapentinnortriptyline combination. Int J Res Orthop 2020;6:1365-70. 